\title{
Neonatal Culture Negative Sepsis, AE
}

National Cancer Institute

\section{Source}

National Cancer Institute. Neonatal Culture Negative Sepsis, AE. NCI Thesaurus. Code C154928.

An adverse event in a newborn characterized by a systemic inflammatory response without identifiable cause. 